\title{
Data-Driven MRSI Spectral Localization Via Low-Rank Component Analysis
}

\author{
Jeffrey Kasten*, Franois Lazeyras, and Dimitri Van De Ville
}

\begin{abstract}
Magnetic resonance spectroscopic imaging (MRSI) is a powerful tool capable of providing spatially localized maps of metabolite concentrations. Its utility, however, is often depreciated by spectral leakage artifacts resulting from low spatial resolution measurements through an effort to reduce acquisition times. Though model-based techniques can help circumvent these drawbacks, they require strong prior knowledge, and can introduce additional artifacts when the underlying models are inaccurate. We introduce a novel scheme in which a generative model is estimated from the raw MRSI data via a regularized variational framework that minimizes the model approximation error within a measurement-prescribed subspace. As additional a priori information, our approach relies only upon a measured field inhomogeneity map at high spatial resolution. We demonstrate the feasibility of our approach on both synthetic and experimental data.
\end{abstract}

Index Terms-Chemical shift imaging, constrained reconstruction, low-rank approximation, magnetic resonance spectroscopic imaging, total variation.

\section{INTRODUCTION}

$\mathbf{S}$ INCE the ability to encode spatial information through the use of magnetic field gradients was introduced nearly 40 years ago, the notion of simultaneous spatial and spectral localization, achieved by magnetic resonance spectroscopic imaging (MRSI), has served as an attractive concept in the field of medical imaging. The ability to noninvasively observe spatial maps of metabolite concentrations, for example, in the human brain, can offer functional, as well as pathological insights, perhaps even before structural aberrations or behavioral symptoms are made manifest (e.g., see [1] and [2]).

Manuscript received March 15, 2013; revised May 14, 2013; accepted May 20, 2013. Date of publication June 04, 2013; date of current version October 02, 2013. This work was supported in part by the Swiss National Science Foundation under Grant SNF 320030-135425, Grant PP00P2-123438, and Grant PP00P2-146318, and in part by the Center for Biomedical Imaging (CIBM). Asterisk indicates corresponding author.

*J. Kasten is with the Institute of Bioengineering, Ecole Polytechnique Fédérale de Lausanne, 1015 Lausanne, Switzerland, and also with the Department of Radiology and Medical Informatics, University of Geneva, 1211 Geneva, Switzerland (e-mail: jeffrey.kasten@epfl.ch).

F. Lazeyras is with the Department of Radiology and Medical Informatics, University of Geneva, 1211 Geneva, Switzerland (e-mail: Francois.Lazeyras@unige.ch).

D. Van De Ville is with the Institute of Bioengineering, Ecole Polytechnique Fédérale de Lausanne, 1015 Lausanne, Switzerland, and also with the Department of Radiology and Medical Informatics, University of Geneva, 1211 Geneva, Switzerland (e-mail: dimitri.vandeville@epfl.ch).

Color versions of one or more of the figures in this paper are available online at http://ieeexplore.ieee.org.

Digital Object Identifier 10.1109/TMI.2013.2266259
The most basic MRSI technique, and that which remains the gold standard, is the chemical shift imaging (CSI) experiment [3], which acquires a free induction decay (FID) at each phaseencoded position in the spatial Fourier domain (henceforth referred to as " $k$-space"), and then reconstructs localized spectral maps via the inverse spatial Fourier transform. This technique, however, suffers from a number of significant drawbacks. Perhaps the foremost is that practical restrictions on the total measurement time, as well as the available signal-to-noise (SNR), in a typical clinical environment limit the number of encodings, and generally only the lowest spatial frequencies (i.e., those typically containing the highest signal energy) are acquired. As the resolution of the reconstructed images is limited by the Fourier pixel size, which cannot adopt an arbitrary shape to match the underlying anatomical structure, the reconstructed maps tend to be rather low resolution, precluding the probing of fine structure. A related consequence is the broad spatial pointspread function (PSF), leading to spectral contamination between spatially distal regions, known as the "spectral leakage" artifact. This results from the implicit assumption underlying the Fourier transform that the object is indeed band-limited to the extent of the acquired measurements, which for most biological samples is increasingly violated as a greater subset of high-frequency encodes is disregarded. Though these PSF effects can be mitigated through additional filtering in the spatial Fourier domain, for example using a Hanning window in order to attenuate the side lobes, such measures are necessarily accompanied by an additional loss of spatial resolution.

Throughout the years, various approaches have been suggested which seek to surmount these limitations. Some of these are based on removing nonsignificant points in $k$-space [4]-[6], employing alternate sampling strategies [7]-[9], using higherorder gradients to adjust the PSF [10], and applying sensitivity encoding (SENSE) [11]. Another approach is through the use of model-based techniques to circumvent the explicit use of the Fourier transform in image reconstruction. In this work, we are primarily interested in extending those techniques falling into this last category.

One of the earliest model-based techniques is spectral localization by imaging (SLIM) [12]. In a SLIM experiment, the observed MR signal is modeled as a sum of signals originating from distinct and spectrally-homogeneous anatomically-defined compartments. Once the compartment geometry has been specified, normally with the aid of additional high-resolution anatomical images, finding the original spectra amounts to solving a linear least squares (LS) problem. A number of extensions to SLIM have since been proposed, including [13], which extends the basic SLIM model as a generalized series, 
and BSLIM [14], which seeks to compensate the estimated spectra for local variations in the static magnetic field $\left(B_{0}\right)$ due to tissue susceptibility. Other recent model-based approaches include [15], [16], which further exploit notions such as sparsity and more flexible basis sets.

One drawback of the original SLIM concept, however, is that it hinges upon the compartmental homogeneity assumption, which is very rarely achieved in practice. Moreover, the notion that the spectrally homogeneous compartment geometry indeed corresponds to the structural anatomy may not be valid, in which case the most effective method for determining the compartments prior to the reconstruction is unclear.

In this work, we continue in the spirit of SLIM, yet proceed without the use of a priori compartment or spectral information. Instead, high-resolution components are estimated directly from the low-resolution MRSI data itself, requiring only a measured local field inhomogeneity map (for example, using the method described in [17]) as an additional imaging modality. Our contribution originates from the notion that despite the relatively low dimensionality of the acquired measurements, the inherent signal often resides within a yet lower-dimensional subspace. In other words, the underlying spatio-spectral distribution admits a low-dimensional representation in a well-chosen basis. There are a burgeoning number of methods within the MR community which seek to explicate the input data by exploiting this very principle, most recently in the field of dynamic imaging [18]-[21]. Similarly, [22] posed the MRSI reconstruction problem as a hierarchical nonnegative matrix factorization, assuming a limited basis set in the temporal frequency domain. The originality of our method lies in the fact that information derived from a high-resolution $B_{0}$ inhomogeneity map is used to circumvent a number of system and sample-dependent factors which tend to thwart or disallow otherwise straightforward low-dimensional representations that are better suited for MRSI reconstruction and interpretation when considering limited sample sets. Moreover, we employ state-of-the-art image regularization to further guide the recovery of high-resolution components. We demonstrate our approach on both synthetic and experimental data.

\section{THEORETICAL BACKGROUND}

\section{A. The SLIM Framework Revisited}

In a standard CSI experiment, the measured signal at $k$-space location $\mathbf{k}_{i}, i=1, \ldots, M$ and time $t$ can be expressed as

$$
s\left(\mathbf{k}_{i}, t\right)=\int_{-\infty}^{\infty} \int_{\mathrm{FOV}} \rho(\mathbf{x}, f) e^{-2 \pi j\left(\mathbf{k}_{i} \cdot \mathbf{x}+f t\right)} d \mathbf{x} d f .
$$

Here, $\rho(\mathbf{x}, f)$ represents the spatio-spectral distribution of the imaged object, where $\mathbf{x}$ specifies position in the spatial domain, $f$ the temporal frequency, and FOV the field-of-view.

The original SLIM concept was impelled by the postulate that the distribution of metabolites of interest is often linked with the distribution of water protons in biological samples. Therefore, a priori knowledge of anatomical features derived from structural ${ }^{1} \mathrm{H}$ MRI could be used to inform the reconstruction of spectroscopic images, an idea which can be considered within a broader framework of "constrained imaging" [23]-[25]. With SLIM, high resolution anatomical MR images are used to define spectrally homogeneous compartments, which can be considered as generalized "voxels," and can assume arbitrary shape to match anatomical structure. This represents a significant departure from standard Fourier imaging, in which the acquisition volume is traditionally limited to be rectangular. The imaged object is modeled as

$$
\rho_{\mathrm{SLIM}}(\mathbf{x}, f)=\sum_{k=1}^{K} q_{k}(f) \chi_{k}(\mathbf{x})
$$

where $q_{k}(f)$ is the spectrum associated with compartment $k$, and the $\chi_{k}(\mathbf{x})$ are indicator functions such that

$$
\chi_{k}(\mathbf{x})=\left\{\begin{array}{lc}
1, & \mathbf{x} \in \text { compartment } k \\
0, & \text { otherwise }
\end{array}\right.
$$

Generally speaking, the $\chi_{k}$ need not be strictly limited to binary indicator functions. In principle, any $\chi_{k}(\mathbf{x}) \in \mathbb{C}$ as may be deemed appropriate for a given application can be used.

The BSLIM model, in which local field inhomogeneities are explicitly taken into account, can be similarly expressed with the addition of a spatially-dependent frequency shift term

$$
\rho_{\text {BSLIM }}(\mathbf{x}, f)=\sum_{k=1}^{K} q_{k}(f-\Delta f(\mathbf{x})) \chi_{k}(\mathbf{x})
$$

where $\Delta f(\mathbf{x})=(\gamma / 2 \pi) \Delta B_{0}(\mathbf{x})$, with $\gamma$ as the gyromagnetic ratio. By inserting the above equation into (1), we obtain

$$
\begin{aligned}
& \int_{-\infty \mathrm{FOV}}^{s_{\mathrm{BSLIM}}\left(\mathbf{k}_{i}, t\right)} \int_{k=1}^{\infty} \sum_{k}^{K} q_{k}(f-\Delta f(\mathbf{x})) \chi_{k}(\mathbf{x}) \cdot e^{-2 \pi j\left(\mathbf{k}_{i} \cdot \mathbf{x}+f t\right)} d \mathbf{x} d f \\
= & \sum_{k=1}^{K} \underbrace{\int_{-\infty}^{\infty} q_{k}(f) e^{-2 \pi j f t} d f}_{Q_{k}(t)} \cdot \underbrace{\int_{\mathrm{FOV}} \chi_{k}(\mathbf{x}) e^{-2 \pi j\left(\mathbf{k}_{i} \cdot \mathbf{x}+\Delta f(\mathbf{x}) t\right)} d \mathbf{x}}_{H_{k}\left(\mathbf{k}_{i}, t\right)} \\
= & \sum_{k=1}^{K} Q_{Q_{k}(t) H_{k}\left(\mathbf{k}_{i}, t\right), \quad i=1, \ldots, M}^{(5)}
\end{aligned}
$$

where $Q_{k}(t)$ is the FID originating from the $k$ th compartment, and $H_{k}\left(\mathbf{k}_{i}, t\right)$ are the so-called BSLIM kernels. Similarly, in the homogenous case $(\Delta f(\mathbf{x})=0)$, the resulting measurements remain separable in $\mathbf{k}_{i}$ and $t$

$$
s_{\mathrm{SLIM}}\left(\mathbf{k}_{i}, t\right)=\sum_{k=1}^{K} Q_{k}(t) K_{k}\left(\mathbf{k}_{i}\right), \quad i=1, \ldots, M
$$

with $K_{k}\left(\mathbf{k}_{i}\right)=H_{k}\left(\mathbf{k}_{i}, 0\right)$. In both cases, the resulting system of linear equations is overdetermined so long as the number of measurements exceeds the number of compartments, which is usually the case in practice, and can be solved via standard LS regression. 
Though extensions to the SLIM framework such as BSLIM add versatility to the basic model, they still adhere to the original assumption that the spatio-spectral distribution should somehow parallel the corresponding anatomy. While this may be a reasonable expectation in some applications, it places a significant restriction on the set of plausible support regions and accordingly on the capacity of the model to uncover the "true" distributions or unexpected solutions. Furthermore, a robust framework for determining the appropriate number of compartments is lacking, especially if the salience of segmentation data from high-resolution scans is contentious. It is therefore natural to ask whether the MRSI data itself may be able to provide some of these basic yet crucial model parameters.

\section{B. MRSI Component Analysis}

We begin with the basic assumption that the data fundamentally yields a low-dimensional bilinear decomposition in the form of (2), yet we have neither a priori access to the compartments, nor to their associated spectra, i.e.,

$$
\hat{\rho}(\mathbf{x}, t)=\sum_{k=1}^{K} u_{k}(\mathbf{x}) v_{k}(t)
$$

when expressed in the time domain for some $u_{k}, v_{k}$, and unknown $K \leq M$. Hence, the acquired measurements can be expressed as

$$
s\left(\mathbf{k}_{i}, t\right)=\left\{\mathcal{F}_{x}\{\hat{\rho}(\mathbf{x}, t)\}\right\}_{\downarrow_{\Omega}}+\eta\left(\mathbf{k}_{i}, t\right), \quad i=1, \ldots, M
$$

where $\mathcal{F}_{x}$ is the spatial Fourier transform, $\{\cdot\}_{\downarrow_{\Omega}}$ represents a spatial downsampling operator which retains those measurements corresponding to the $\left\{\mathbf{k}_{i}\right\}_{i=1}^{M}$ prescribed by the chosen $k$-space trajectory, and $\eta\left(\mathbf{k}_{i}, t\right)$ is additive measurement noise (which we assume to be i.i.d. Gaussian).

1) Inhomogeneity Compensation: It should be clear from expressions (3)-(5) that the existence of local field inhomogeneities tends to impede the separability of the basic model (7). Though separable solutions could be found given a suitable decomposition of $s\left(\mathbf{k}_{i}, t\right)$, for example using the singular value decomposition (SVD), they would necessarily reflect any inhomogeneity-induced perturbations.

To compensate, an additional measurement of the field inhomogeneity is therefore necessary, primarily reflecting the sample-specific magnetic susceptibilities. One key element is that the spatial resolution of this map can be arbitrarily chosen (within measurement system limitations), and is usually acquired at the higher resolution of the structural images (e.g., matrix size $128 \times 128$ ). Having direct access to this information, we introduce an operator $\mathscr{P}_{\Delta f}$, acting in the high-resolution space, whose effective action is to shift a spatio-spectral volume along the temporal frequency axis in accordance with $\Delta f(\mathbf{x})$. As this operator is ultimately discretized, it would be beneficial at this point to refer to the discretized versions of the various continuous-domain functions, notations for which are summarized in Table I. Hence, (7) becomes

$\hat{\rho}\left[\mathbf{x}_{n}, t_{m}\right]=\sum_{k=1}^{K} u_{k}\left[\mathbf{x}_{n}\right] v_{k}\left[t_{m}\right], n=1, \ldots, N, m=1, \ldots, T$
TABLE I

VARIOUS SAMPLING GRIDS AND NOTATIONS USED BY THE RECONSTRUCTION ROUTINE

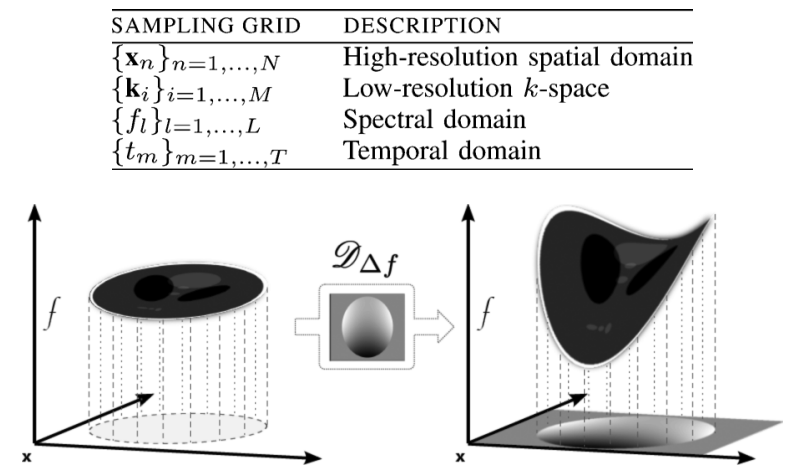

Fig. 1. Effect of the spectral deformation operator on a slice of a generic spatiospectral volume.

or in matrix form as

$$
\mathbf{P}=\mathbf{U V}
$$

where $\mathbf{U} \in \mathbb{C}^{N \times K}, \mathbf{V} \in \mathbb{C}^{K \times T}$.

Definition 1: Given a spatial inhomogeneity profile, $\Delta f(\mathbf{x})$, the spectral deformation operator, $\mathscr{V}_{\Delta f}$, acting upon a spatiospectral volume, $\varphi$, is given by

$$
\mathscr{P}_{\Delta f}\{\varphi\}:=\varphi(\mathbf{x}, f) \delta(f-\Delta f(\mathbf{x})) .
$$

The above definition holds discretely given a suitable interpolant in the temporal frequency domain, though $\mathcal{O}_{\Delta f}$ can also be applied in the temporal domain, such that $\mathscr{V}_{\Delta f}\left\{\mathcal{F}_{t}^{-1}\{\varphi\}\right\}=\mathcal{F}_{t}^{-1}\{\varphi\}(\mathbf{x}, t) e^{-2 \pi j \Delta f(\mathbf{x}) t}$, where $\mathcal{F}_{t}^{-1}$ is the inverse temporal Fourier transform. The effect of $\mathscr{V}_{\Delta f}$ on a slice of a generic volume, $\varphi\left(\mathbf{x}, f_{0}\right)$, is illustrated in Fig. 1. With all the operators defined, the discrete set of measurements for a given CSI experiment can be expressed as

$$
\mathbf{S}=\left\{\mathcal{F}_{x}\left\{\mathscr{P}_{\Delta f}\{\mathbf{U V}\}\right\}\right\}_{\downarrow_{\Omega}}+\mathbf{N}
$$

with $\mathbf{S}, \mathbf{N} \in \mathbb{C}^{M \times T}$, which reduces to (the discretized version of) (8) when $\Delta f(\mathbf{x})=0$.

2) Recovery of High-Resolution Components: Ideally, we would like to obtain reconstructions at similar resolutions to those of standard structural imaging. This was achieved within the SLIM/BSLIM framework by assuming an a priori knowledge of the high-resolution compartment geometry. In the absence of such information, and thereby remaining limited to the low-resolution measurements (and an acquired field map), the same reconstruction problem becomes ill-posed. Furthermore, to the authors' knowledge, there is no globally-convergent method of simultaneously solving for the individual maps and spectra, $u_{k}$ and $v_{k}$, respectively. Nonetheless, we proceed by formulating the reconstruction as a minimization over a cost function, $\mathcal{J}$, consisting of a data fidelity term over the acquired $k$-space measurements, and a regularization term based upon the total variation (TV) semi-norm, which is classically defined as

$$
\operatorname{TV}\{\zeta\}=\int_{\mathcal{D}}|\nabla \zeta(\mathbf{x})| d \mathbf{x}
$$


where $\mathcal{D}$ is the image domain. TV has recently gained notoriety within the MR community due to its versatility in addressing a wide array of problems such as denoising [26], suppression of truncation artifacts/deblurring [27], [28], inpainting for sensitivity maps [29], and compressed sensing [30]. Much of the allure of TV rests in its proclivity for removing noise-like artifacts, while preserving sharp edges. However, it has been shown that use of the TV semi-norm ( $L_{1}$ norm of the gradient) results in images exhibiting staircase-like artifacts, which are visually displeasing or physically implausible in the case of MRI [31]. This is due to the fact that the above TV penalty promotes images with sparse gradients, i.e., it often leads to piecewise constant solutions. Recently, numerous techniques have been proposed which address this issue, for example, by considering higher-order functionals [32]-[35]. In this work, we adopt the method of [32], referred to as the total generalized variation of second order $\left(\mathrm{TGV}^{2}\right)$, which offers a compromise between penalizing on the first and second derivatives, where

$$
\operatorname{TGV}_{\alpha}^{2}\{\zeta\}=\min _{h} \alpha_{1} \int_{\mathcal{D}}|\nabla \zeta(\mathbf{x})-h| d \mathbf{x}+\alpha_{0} \int_{\mathcal{D}}|\mathcal{E}\{h\}| d \mathbf{x}
$$

or its discrete analog

$$
\begin{array}{r}
\operatorname{TGV}_{\alpha}^{2}\{z\}=\min _{h} \alpha_{1}\left\|\nabla z\left[\mathbf{x}_{n}\right]-h\right\|_{\ell_{1}}+\alpha_{0}\|\mathcal{E}\{h\}\|_{\ell_{1}}, \\
n=1, \ldots, N
\end{array}
$$

and $\mathcal{E}\{u\}=\left(\nabla u+\nabla u^{T}\right) / 2$ denotes the symmetrized derivative. In our experiments, the discretized gradient operator is realized using forward finite differences with Neumann boundary conditions. Following the authors' suggestion in [32], in our experiments $\alpha_{0}$ and $\alpha_{1}$ were chosen such that $\alpha_{0}=2 \alpha_{1}$. With these ingredients in place, the reconstruction can be posed as

$$
\min \mathcal{J}=\left\|\left\{\mathcal{F}_{x}\left\{\mathscr{O}_{\Delta f}\{\mathbf{U V}\}\right\}\right\}_{\downarrow_{s}}-\mathbf{S}\right\|_{F}^{2}+\mu \mathrm{TGV}_{\alpha}^{2}\{\mathbf{U}\}
$$

where $\mu$ is a constant that controls the regularization strength, and $\|\cdot\|_{F}$ is the Frobenius norm.

At this point, a few words seeking to explicate the assumptions behind the $u_{k}$ and $v_{k}$ would be beneficial. Firstly, though written as a function of $\mathbf{x}_{n}$ alone in (9), the $u_{k}$ can also be considered as having a temporal dimension, but which remains constant in time so that $u_{k}\left[\mathbf{x}_{n}\right]=u_{k}\left[\mathbf{x}_{n}, t_{m}\right]=u_{k}\left[\mathbf{x}_{n}, t_{1}\right] \forall m=$ $1, \ldots, T$. This can also be seen by considering the vectorized version of (10)

$$
\begin{aligned}
\operatorname{vec}(\mathbf{P}) & =\left(\mathbf{I}^{(T \times T)} \otimes \mathbf{U}\right) \operatorname{vec}(\mathbf{V}) \\
& =\left(\mathbf{V}^{\mathrm{T}} \otimes \mathbf{I}^{(K \times K)}\right) \operatorname{vec}(\mathbf{U})
\end{aligned}
$$

where $\otimes$ denotes the Kronecker product, $(\cdot)^{\mathrm{T}}$ the matrix transpose, and $\operatorname{vec}(\mathbf{A}) \in \mathbb{C}^{(m n \times 1)}$ is defined by stacking the columns of a matrix $\mathbf{A} \in \mathbb{C}^{(m \times n)}$. The later operation can be similarly extended for higher-dimensional arrays, such that for $\mathbf{A} \in \mathbb{C}^{(m \times n \times p)}, \operatorname{vec}(\mathbf{A}) \in \mathbb{C}^{(m n p \times 1)}$, and so forth. For ease of notation, we will henceforth denote vectorized versions of matrices and multi-dimensional arrays by their corresponding boldface lowercase letters and a $(\vec{\cdot})$, such that $\operatorname{vec}(\mathbf{A})=\overrightarrow{\mathbf{a}}$. The interpretation in (17) is noteworthy because it allows $\mathscr{V}_{\Delta f}$, a spatio-spectral operator, to act upon $\mathbf{U}$ alone, considered as a spatio-spectral volume, which in turn allows us to isolate
$\mathbf{U}$ (and hence $\mathbf{V}$ ) during the minimization. It is, in fact, this time constancy which ensures the bilinear structure of (9) and (10), hence maintaining separability amounts to constraining the $u_{k}$ to lie on the $f=0$ plane in the temporal frequency domain. Secondly, in order to endow the spatial components with some physical significance, and to better frame the TGV regularization, we require the $u_{k}$ to be real-valued and nonnegative, i.e., $u_{k} \in \mathbb{R}^{+}$. We further on refer to the intersection of the nonnegative and time-constant constraint sets as the set $\mathbb{E}$. The formulation of (17) can be easily updated for general $u_{k}\left[\mathbf{x}_{n}, t_{m}\right]$ (i.e., nonconstant in the temporal dimension). Letting $\mathrm{U}\left[t_{m}\right]=\left(u_{1}\left[\mathbf{x}_{n}, t_{m}\right] u_{2}\left[\mathbf{x}_{n}, t_{m}\right] \cdots u_{K}\left[\mathbf{x}_{n}, t_{m}\right]\right)$ for $m=1, \ldots, T$

$$
\begin{aligned}
\overrightarrow{\mathbf{p}} & =\left(\begin{array}{cccc}
\mathrm{U}\left[t_{1}\right] & \mathbf{0}^{(N \times K)} & \cdots & \mathbf{0}^{(N \times K)} \\
\mathbf{0}^{(N \times K)} & \mathrm{U}\left[t_{2}\right] & \cdots & \mathbf{0}^{(N \times K)} \\
\vdots & \vdots & \ddots & \vdots \\
\mathbf{0}^{(N \times K)} & \mathbf{0}^{(N \times K)} & \cdots & \mathrm{U}\left[t_{T}\right]
\end{array}\right) \overrightarrow{\mathbf{v}}=\boldsymbol{\Gamma} \overrightarrow{\mathbf{v}} \\
& =\left(\mathbf{V}^{\mathrm{T}} \otimes \mathbf{I}^{(K \times K)}\right) \overrightarrow{\mathbf{u}}=\mathbf{\Xi} \overrightarrow{\mathbf{u}} .
\end{aligned}
$$

Though no explicit assumptions are made with regards to the expected form of the $v_{k}$, for example concerning the degree of sparsity or adherence to Lorentzian or Gaussian line shapes, we do restrict the entries of $\mathbf{V}$ such that $\|\mathbf{V}\|_{F} \leq 1$, essentially enabling the dictionary to depreciate nonessential elements [36]-[38]. Finally, incorporating the stated constraints, the reconstruction is given by

$$
\begin{aligned}
& \min \mathcal{J}=\left\|\boldsymbol{\Omega} \mathbf{F D} \Xi \overrightarrow{\mathbf{u}}_{\mathfrak{R}}-\overrightarrow{\mathbf{s}}\right\|_{2}^{2}+\mu \mathrm{TGV}_{\alpha}^{2}\left\{\overrightarrow{\mathbf{u}}_{\mathfrak{R}}\right\}, \\
& \text { s.t. } \quad \overrightarrow{\mathbf{u}}_{\mathfrak{R}} \in \mathbb{E},\|\overrightarrow{\mathbf{v}}\|_{2} \leq 1
\end{aligned}
$$

where $\boldsymbol{\Omega}, \mathbf{F}$, and $\mathbf{D}$ are the equivalent matrices for operators $\{\cdot\}_{\downarrow_{\Omega}}, \mathcal{F}_{x}$, and $\mathcal{V}_{\Delta f}$, respectively, and $\overrightarrow{\mathbf{u}}_{\mathfrak{R}}=2 \operatorname{Re}\left\{\overrightarrow{\mathbf{u}}+\overrightarrow{\mathbf{u}}^{*}\right\}$, where $(\cdot)^{*}$ denotes the complex conjugate. In order to solve (20), we adopt an Augmented Lagrangian (AL) approach, otherwise known as the method of multipliers (e.g., see [39]), in which an alternating minimization is performed over a set of surrogate variables. We first write (20) as the constrained optimization problem

$$
\begin{array}{ll}
\min & \mathcal{J}=\left\|\boldsymbol{\Omega} \mathbf{F D} \Xi \overrightarrow{\mathbf{u}}_{\Re}-\overrightarrow{\mathbf{s}}\right\|_{2}^{2}+\mu \mathrm{TGV}_{\alpha}^{2}\{\overrightarrow{\mathbf{r}}\} \\
\text { s.t. } & \overrightarrow{\mathbf{u}}_{\mathfrak{R}} \in \mathbb{E}, \overrightarrow{\mathbf{r}}=\overrightarrow{\mathbf{u}}_{\mathfrak{R}},\|\overrightarrow{\mathbf{v}}\|_{2} \leq 1
\end{array}
$$

which we then solve using the AL formulation

$$
\begin{aligned}
\min \mathcal{L}= & \left\|\boldsymbol{\Omega} \mathbf{F D} \Xi \overrightarrow{\mathbf{u}}_{\mathfrak{R}}-\overrightarrow{\mathbf{s}}\right\|_{2}^{2}+\mu \mathrm{TGV}_{\alpha}^{2}\{\overrightarrow{\mathbf{r}}\} \\
& +\beta\left\|\overrightarrow{\mathbf{u}}_{\mathfrak{R}}-\overrightarrow{\mathbf{r}}\right\|_{2}^{2} \\
& +\left\langle\overrightarrow{\boldsymbol{\lambda}}, \overrightarrow{\mathbf{u}}_{\mathfrak{R}}-\overrightarrow{\mathbf{r}}\right\rangle, \quad \text { s.t. } \overrightarrow{\mathbf{u}}_{\mathfrak{R}} \in \mathbb{E},\|\overrightarrow{\mathbf{v}}\|_{2} \leq 1
\end{aligned}
$$

where $\vec{\lambda}$ is an auxiliary variable whose role is similar to that of a Lagrange multiplier, $\beta$ is the "penalty coefficient," and $\langle\cdot\rangle$ denotes an inner product. Here, we propose a four-step scheme for solving (22)

$$
\begin{aligned}
\overrightarrow{\mathbf{u}}_{\Re, n+1}= & \arg \min _{\overrightarrow{\mathbf{u}}_{\mathfrak{R}} \in \mathbb{E}}\left\|\boldsymbol{\Omega F D} \Xi_{n} \overrightarrow{\mathbf{u}}_{\mathfrak{R}}-\overrightarrow{\mathbf{s}}\right\|_{2}^{2} \\
& +\beta\left\|\overrightarrow{\mathbf{u}}_{\mathfrak{R}}-\left(\overrightarrow{\mathbf{r}}_{n}-\frac{\overrightarrow{\boldsymbol{\lambda}}_{n}}{\beta}\right)\right\|_{2}^{2} \\
\overrightarrow{\mathbf{v}}_{n+1}= & \arg \min _{\|\overrightarrow{\mathbf{v}}\|_{2} \leq 1}\left\|\boldsymbol{\Omega} \mathbf{F D} \Xi \overrightarrow{\mathbf{u}}_{\mathfrak{R}, n+1}-\overrightarrow{\mathbf{s}}\right\|_{2}^{2}
\end{aligned}
$$




$$
\begin{aligned}
& \overrightarrow{\mathbf{r}}_{n+1}=\arg \min _{\overrightarrow{\mathbf{r}}}\left\|\overrightarrow{\mathbf{u}}_{\Re, n+1}-\left(\overrightarrow{\mathbf{r}}-\frac{\overrightarrow{\boldsymbol{\lambda}}_{n}}{\beta}\right)\right\|_{2}^{2}+\frac{\mu}{\beta} \operatorname{TGV}_{\alpha}^{2}\{\overrightarrow{\mathbf{r}}\} \\
& \overrightarrow{\boldsymbol{\lambda}}_{n+1}=\overrightarrow{\boldsymbol{\lambda}}_{n}+\beta\left(\overrightarrow{\mathbf{u}}_{\Re, n+1}-\overrightarrow{\mathbf{r}}_{n+1}\right) .
\end{aligned}
$$

The $\overrightarrow{\mathbf{r}}$ subproblem (25) is referred to as the total (generalized) variation denoising problem, whose solution can be obtained using the primal-dual method originally proposed in [32]. To solve the $\overrightarrow{\mathbf{u}}_{\mathfrak{R}}$ subproblem, we use a projected gradient approach, whereby at each internal iteration, $l, \overrightarrow{\mathbf{u}}_{\Re}$ is updated as

$$
\overrightarrow{\mathbf{u}}_{\mathfrak{R}, l+1}=\mathcal{P}_{C}\left(\overrightarrow{\mathbf{u}}_{\mathfrak{R}, l}-\frac{1}{L} \nabla \mathcal{L}\left(\overrightarrow{\mathbf{u}}_{\mathfrak{R}, l}\right)\right)
$$

where $L$ is an appropriate upper bound on the Lipschitz constant of the gradient, and $\mathcal{P}_{C}$ is the Euclidean projector onto the set $C$, which in our case $(C=\mathbb{E})$ amounts to projecting the solution onto the $f=0$ plane, followed by the projection $\mathcal{P}_{\mathbb{R}^{+}}(x)=$ $\max \{0, x\}$. The gradient, $\nabla \mathcal{L}\left(\overrightarrow{\mathbf{u}}_{\mathfrak{R}, l}\right)$ is given by

$$
\begin{aligned}
\nabla \mathcal{L}\left(\overrightarrow{\mathbf{u}}_{\mathfrak{R}, l}\right) & =2 \operatorname{Re}\left\{\left(\boldsymbol{\Xi}^{H} \mathbf{D}^{H} \mathbf{F}^{H} \boldsymbol{\Omega}^{H} \mathbf{\Omega} \mathbf{F} \mathbf{D}+\beta \mathbf{I}\right) \overrightarrow{\mathbf{u}}_{l}\right. \\
& \left.-\boldsymbol{\Xi}^{H} \mathbf{D}^{H} \mathbf{F}^{H} \boldsymbol{\Omega}^{H} \overrightarrow{\mathbf{s}}-\beta\left(\overrightarrow{\mathbf{r}}-\frac{\vec{\lambda}}{\beta}\right)\right\}
\end{aligned}
$$

where $(\cdot)^{H}$ represents the Hermitian transpose and $\mathbf{I}$ the identity matrix. For completeness, the adjoint operations $\mathbf{\Omega}^{H}$ and $\mathbf{D}^{H}$, can be characterized as a zero-filling, and an appeal to Definition 1 with a flip of sign, respectively. Also worth mentioning is that the above forward and adjoint matrices need not be explicitly constructed in practice, as the associated operations may be applied point-wise along the temporal axis. Finally, the convergence speed of (23) may be significantly improved by employing a fast projected gradient scheme such as [28], [40].

A solution to the $\overrightarrow{\mathbf{v}}$ subproblem may be found after a substitution of (18) into (24)

$$
\overrightarrow{\mathbf{v}}_{n+1}=\arg \min _{\|\overrightarrow{\mathbf{v}}\|_{2} \leq 1}\|\boldsymbol{\Omega F D \Gamma} \overrightarrow{\mathbf{v}}-\overrightarrow{\mathbf{s}}\|_{2}^{2}
$$

which can be also solved using projected gradient methods, where the projection step is given by

$$
\mathcal{P}(x)=\left\{\begin{array}{ll}
\frac{x}{\|x\|_{2}}, & \|x\|_{2} \geq 1 \\
x, & \text { otherwise }
\end{array} .\right.
$$

One advantage to using the AL framework is that unlike traditional penalty methods, $\beta$ does not need to grow very large in order to establish equivalence between (20) and (22). Nonetheless, we employ a continuation strategy, steadily increasing $\beta$ in an outer loop once convergence has been reached over the alternating minimization routine, in order to hasten convergence.

\section{MATERIALS AND METHODS}

\section{A. Synthetic Data}

In order to validate the proposed method, we examine reconstructions made available through the use of synthetic data. For these experiments, a numerical phantom was utilized consisting of three compartments (Fig. 2), using a square FOV $([-0.5 \ldots 0.5] \times[-0.5 \ldots 0.5])$ and a $128 \times 128$ Cartesian sampling grid. An inhomogeneity map was also simulated at
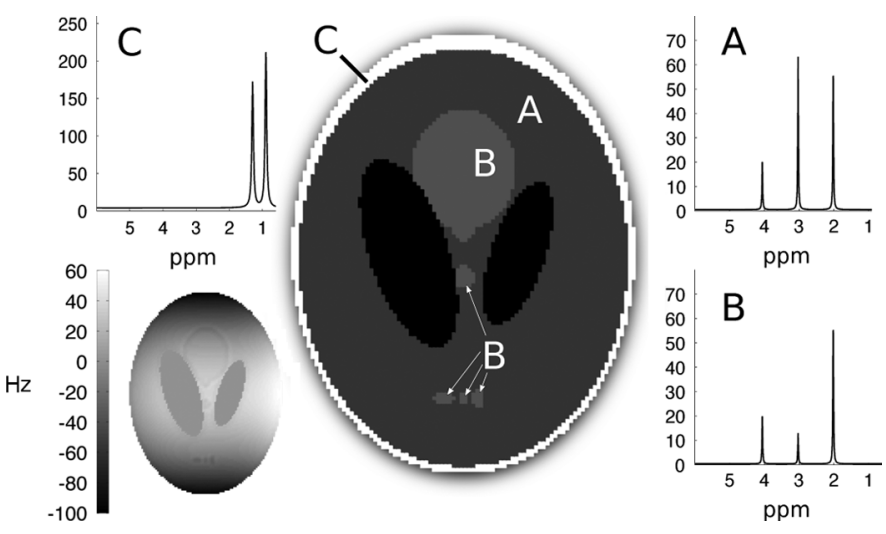

Fig. 2. Synthetic phantom composition and simulated static field inhomogeneity map (lower left).

the same resolution, using a fourth-degree polynomial model along each Cartesian dimension, which was then thresholded by the combined spatial support of the compartments. To emulate changes in magnetic susceptibility between the compartments, additional Laplacian-of-Gaussian filtered images (Gaussian kernel full width at half maximum: three pixel units) for each compartment were added to the final $B_{0}$ map. To each compartment, a unique spectrum with fixed temporal resolution $(T=1024)$ was associated. The final synthetic dataset was obtained by constructing the kernels for each compartment, $H_{k}\left[\mathbf{k}_{i}, t_{m}\right]$ [see (4)] from the high-resolution compartment images and the $B_{0}$ map, and multiplying by the corresponding FID. The measurement process was simulated by selecting the central phase encodes of the resulting dataset, which we took to be a $32 \times 32$ Cartesian sampling grid in $k$-space, and adding Gaussian white noise to the simulated MRSI data. To examine the robustness of the reconstruction to various noise conditions, five realizations for each of three noise scenarios were generated, corresponding to mean SNR values of 13.98, 10.02, and $7.03 \mathrm{~dB}$, to which we further refer as Case 1, Case 2, and Case 3, respectively.

\section{B. Phantom MRSI Data}

To test the performance of our algorithm using actual MR scanner data, we acquired two CSI datasets on a 3.0T Siemens Trio (Siemens Healthcare, Erlangen, Germany), using a twocompartment phantom (Fig. 3). The inner compartment (A) consists of a sphere $($ diameter $=8.7 \mathrm{~cm})$, containing a solution of 50 $\mathrm{mmol} / \mathrm{L} \mathrm{N}$-acetyl-aspartate (NAA) and $50 \mathrm{mmol} / \mathrm{L}$ creatine $(\mathrm{Cr})$ in doped water. The outer cylindrical compartment (B) (height = $13.5 \mathrm{~cm}$, diameter $=10.5 \mathrm{~cm}$ ) is filled with corn oil. Single-voxel spectra were acquired for each compartment using a PRESS sequence (voxel size $=15 \times 15 \times 15 \mathrm{~mm}$, TR $=1700 \mathrm{~ms}$, $\mathrm{TE}=288 \mathrm{~ms}$, bandwidth $=2 \mathrm{kHz}$ ). As a brief caveat, the NAA and $\mathrm{Cr}$ resonances in the inner compartment PRESS spectrum [Fig. 3(A)] should display roughly equal peak heights. Though the integrated peak areas are indeed equivalent, the amplitude discrepancy is due to variations in linewidth. Water-suppressed CSI data were acquired from a $10 \mathrm{~mm}$ slice thickness positioned at the center of the inner compartment $(\mathrm{FOV}=160 \times 160 \mathrm{~mm}$, $\mathrm{TR}=1700 \mathrm{~ms}, \mathrm{TE}=288 \mathrm{~ms}$, bandwidth $=1.5 \mathrm{kHz}$, number of FID sampling points $=1024$ ) using both $32 \times 32$ and $64 \times 64$ 


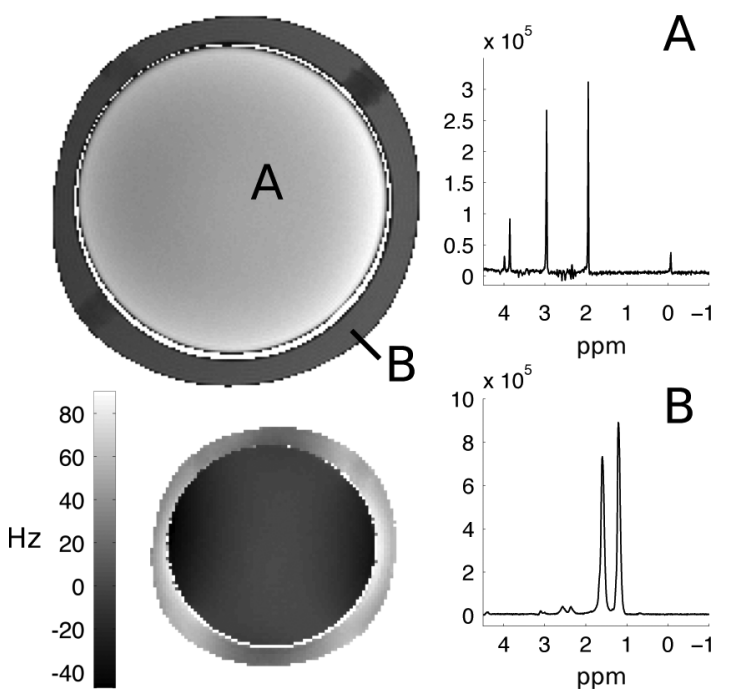

Fig. 3. ${ }^{1} \mathrm{H}$ Phantom composition and acquired static field inhomogeneity map (lower left).

sampling grids. During the same scanning session, a map of local static $B_{0}$ field variations was estimated by acquiring two spoiled gradient echo sequences corresponding to the FOV of the CSI (grid size $=128 \times 128$ ), each chosen with different echo times such that their difference, $\triangle \mathrm{TE}$, ensured that the water and fat resonances were in phase. Though two different CSI grid sizes were acquired, reconstructions were performed only using the lower resolution $(32 \times 32)$ data.

Prior to reconstruction, an initial estimation must be made for the dimensionality of the working subspace, $K_{0}$. For both the synthetic and ${ }^{1} \mathrm{H}$ phantom datasets, $K_{0}$ was determined from the raw measurement matrix, $\mathbf{S} \in \mathbb{C}^{(M \times T)}$, using the method of [41]. Though a detailed exposition is beyond the scope of this paper, the method effectively parametrizes a conjugate prior distribution on the set of bases, and selects the dimensionality of the probabilistic PCA model that maximizes the Bayesian evidence. Ultimately, as $K_{0}$ is selected based upon the inhomogeneitycorrupted measurements, we consider it as an upper-bound on the true underlying data dimensionality, $K$, such that $K_{0} \geq K$. In our case, $K_{0}$ was chosen as 25 and 32 for the synthetic and ${ }^{1} \mathrm{H}$ phantom datasets, respectively. For the synthetic data, the regularization parameter, $\mu$, was determined empirically to minimize the PSNR between the reconstructed data and the ground truth, allowing us to find an $\epsilon$ such that $\left\|\Omega \mathcal{F} \mathscr{Q}_{\Delta f}\{\mathbf{U V}\}-\mathbf{S}\right\|_{F} \leq$ $\epsilon\|\mathbf{S}\|_{F}$. In our experiments, we found for Case 1 an $\epsilon=0.067$ ( $\epsilon=0.163$ and $\epsilon=0.314$ for Case 2 and Case 3, respectively), which was then in turn used to compute $\mu$ for the ${ }^{1} \mathrm{H}$ phantom dataset. In all scenarios, qualitatively and quantitatively superior reconstructions were obtained when initializing $\mathbf{U}$ and $\mathbf{V}$ as random matrices, as opposed to, say, the first $K_{0}$ components resulting from a SVD of the adjoint solution (see below). All computations were carried out in MATLAB 8 (The Mathworks Inc., Natick, MA, USA) on an Intel Xeon $3.33 \mathrm{GHz}$ six-core processor under Mac OSX 10.8.2 with 32 GB RAM. For the presented experimental data, computation times ranged between 100-130 min, though significant speedups are anticipated upon migrating to GPU-based implementations.

\section{RESUlTS}

\section{A. Synthetic Data}

Representative spectra (Case 1) from each reconstruction method are displayed in Fig. 4. The reconstruction in column II was generated by sequentially applying each of the adjoint operators, $\boldsymbol{\Omega}^{H}, \mathbf{F}^{H}$, and $\mathbf{D}^{H}$ to the raw MRSI measurements. For quantitative evaluation, each of the given spectra, as well as the reconstructed spatio-spectral volume as a whole, was compared to the ground truth data. The resulting mean PSNR values across realizations for each SNR scenario are collected in Table II. Following reconstructions, the SVD was computed for representative datasets in each case; the resulting singular value spectra are provided in Fig. 6(a)-(c).

\section{B. Phantom MRSI Data}

As with the synthetic case, a few representative spectra reconstructed by each method are shown in Fig. 5. In this case, the $64 \times 64$ reference CSI data has replaced the adjoint reconstructions presented in Fig. 4. The final singular value spectrum is given in Fig. 6(d).

\section{Discussion}

\section{A. Synthetic Data}

The limitations associated with MRSI reconstructions by low-resolution inverse Fourier transform are clearly visible in Fig. 4 (I). The spectral leakage artifacts, while expectedly severe near the compartment interface $[\mathrm{I}(\mathrm{d})]$, propagate even through the FOV center $[\mathrm{I}(\mathrm{b})]$, demonstrating the profound effects of the PSF. Concomitant lineshape distortions and spectral shifts are also apparent throughout the reconstructed data. Similarly, though the adjoint reconstruction (II) effectively compensates for the inhomogeneity-induced shifts, it is unable to exploit the full high-resolution information from the field map, and to mitigate the PSF effects. In contrast, reconstructions using the proposed method (III and IV) best approximate the ground truth spatio-spectral distribution, offering vastly improved spatial localization and spectral lineshapes. Though the merits of the TGV penalty can be clearly recognized via improved spectral quality in IV(a), IV(b), and markedly reduced spectral leakage from the outer-most compartment $[\operatorname{IV}(c), \operatorname{IV}(d)]$, it can introduce slight partial-volume effects, which are most pronounced around areas containing differing spectral signatures which are small in comparison to the nominal CSI voxel size [IV(c) versus III(c)]. Nonetheless, Table II shows that by and large, the TGV regularization provides the best reconstruction quality by abating the most degrading influences.

\section{B. Phantom MRSI Data}

Looking to the ${ }^{1} \mathrm{H}$ MRSI phantom data (Fig. 5), one notices many of the same artifacts in the standard Fourier reconstruction (I) that were also prevalent in the synthetic case, namely, those due to spectral leakage, especially near the compartment interface. Spectral shifts and lineshape distortions due to the static field inhomogeneity (whose profile is shown in Fig. 3) are also readily seen. For comparison, corresponding spectra taken from the $64 \times 64$ Fourier reconstructed data are shown 
TABLE II

Synthetic Dataset Mean PSNR Values Plus Standard Deviations for the Selected Spectra and Full Reconstructed Datasets (dB). Final Row Reflects Mean Values Over all Voxels Across Realizations

\begin{tabular}{|c|c|c|c|c|c|c|c|c|c|c|c|c|}
\hline \multirow{4}{*}{ SPECTRUM } & \multicolumn{12}{|c|}{$\begin{array}{l}\text { RECONSTRUCTION METHOD } \\
\end{array}$} \\
\hline & \multicolumn{3}{|c|}{ IFFT } & \multicolumn{3}{|c|}{ ADJOINT } & \multicolumn{3}{|c|}{ PROPOSED (NO TGV) } & \multicolumn{3}{|c|}{ PROPOSED } \\
\hline & Case 1 & Case 2 & Case 3 & Case 1 & Case 2 & Case 3 & Case 1 & Case 2 & Case 3 & Case 1 & Case 2 & Case 3 \\
\hline & $17.55 \pm 0.02$ & $17.33 \pm 0.04$ & $17.04 \pm 0.05$ & $21.60 \pm 0.03$ & $21.04 \pm 0.08$ & $20.34 \pm 0.06$ & $38.21 \pm 3.28$ & $31.42 \pm 4.84$ & $26.56 \pm 1.22$ & $41.99 \pm 3.23$ & $37.85 \pm 1.93$ & $35.68 \pm 1.68$ \\
\hline (b) & $15.01 \pm 0.03$ & $14.71 \pm 0.05$ & $14.25 \pm 0.04$ & $21.12 \pm 0.07$ & $20.17 \pm 0.09$ & $18.72 \pm 0.04$ & $35.31 \pm 1.80$ & $29.27 \pm 0.71$ & $24.89 \pm 0.67$ & $36.62 \pm 1.10$ & $32.91 \pm 0.39$ & $30.41 \pm 0.19$ \\
\hline (c) & $17.61 \pm 0.04$ & $17.30 \pm 0.07$ & $16.89 \pm 0.07$ & $21.22 \pm 0.04$ & $20.52 \pm 0.05$ & $19.75 \pm 0.06$ & $36.53 \pm 2.02$ & $27.97 \pm 1.87$ & $27.07 \pm 1.50$ & $36.30 \pm 2.59$ & $32.29 \pm 1.81$ & $30.51 \pm 1.39$ \\
\hline (d) & $18.30 \pm 0.04$ & $18.14 \pm 0.04$ & $17.86 \pm 0.04$ & $20.82 \pm 0.03$ & $20.54 \pm 0.04$ & $20.04 \pm 0.05$ & $38.13 \pm 1.57$ & $33.84 \pm 2.09$ & $30.10 \pm 1.72$ & $40.98 \pm 3.16$ & $39.04 \pm 2.44$ & $36.62 \pm 1.51$ \\
\hline (e) & $18.32 \pm 0.00$ & $18.31 \pm 0.01$ & $18.30 \pm 0.01$ & $24.64 \pm 0.01$ & $24.62 \pm 0.03$ & $24.56 \pm 0.01$ & $48.27 \pm 2.01$ & $41.69 \pm 2.75$ & $38.55 \pm 2.42$ & $48.29 \pm 4.11$ & $46.31 \pm 0.82$ & $44.45 \pm 1.19$ \\
\hline (f) & $19.50 \pm 0.00$ & $19.49 \pm 0.01$ & $19.47 \pm 0.01$ & $23.39 \pm 0.01$ & $23.37 \pm 0.01$ & $23.33 \pm 0.03$ & $51.67 \pm 1.20$ & $45.57 \pm 0.68$ & $40.47 \pm 1.73$ & $48.79 \pm 1.83$ & $47.92 \pm 0.63$ & $43.99 \pm 1.91$ \\
\hline FULL & & & & $20.61 \pm 0.00$ & $18.26 \pm 0.00$ & $16.41 \pm 0.00$ & $44.88 \pm 4.85$ & $31.77 \pm 0.27$ & $27.21 \pm 0.26$ & $48.99 \pm 1.04$ & $43.81 \pm 1.29$ & $38.35 \pm 4.21$ \\
\hline
\end{tabular}

I

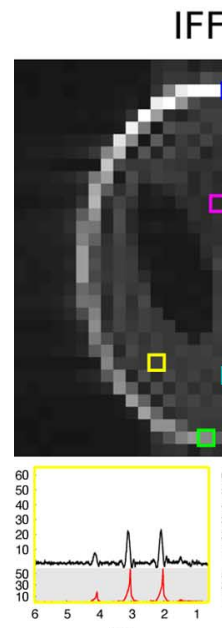

(a)

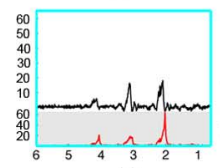

(c)

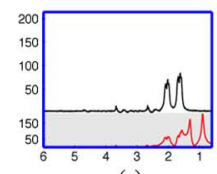

(e)
II

Adjoint
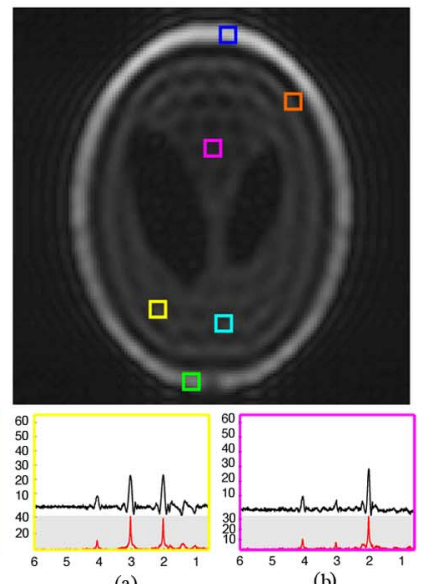

(a)

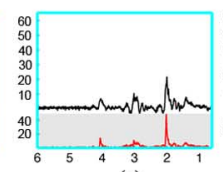

(c)

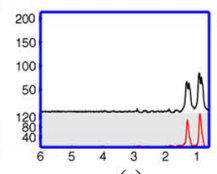

(e)
III
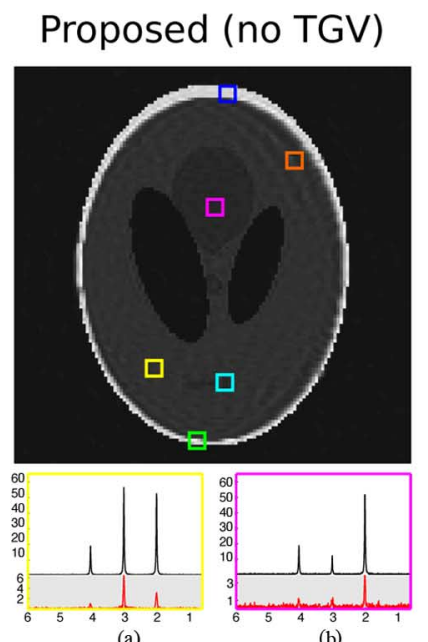

(a)

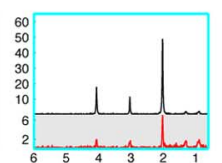

(c)

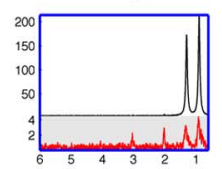

(e)

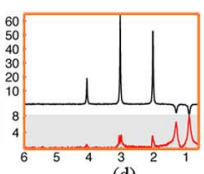

(d)

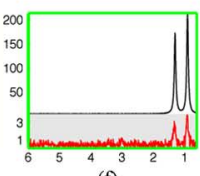

(f)
IV
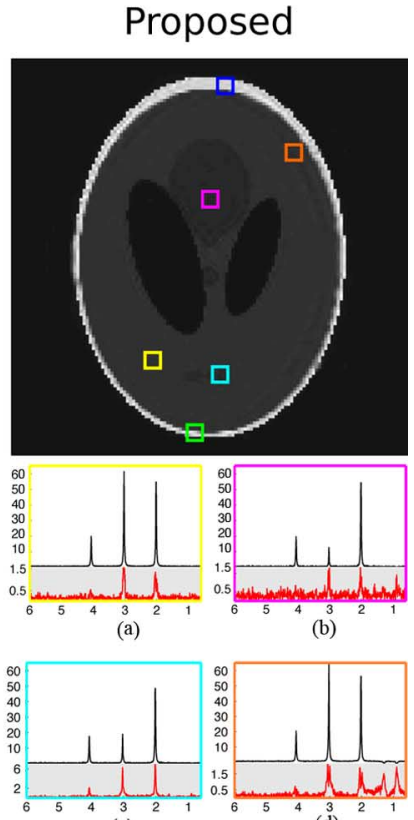

(c)

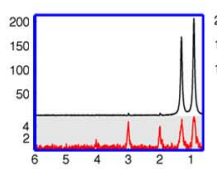

(e)

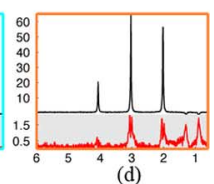

(d)

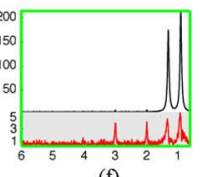

(f)

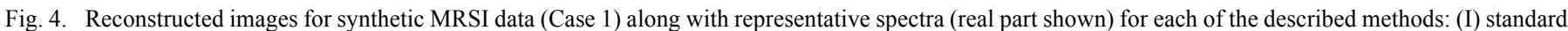

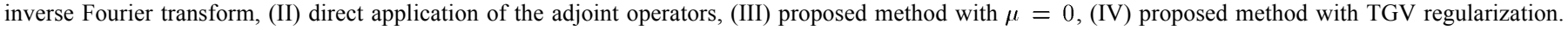

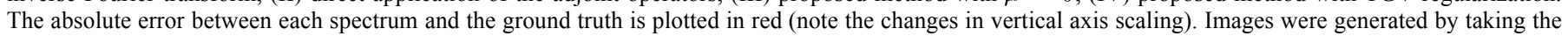
magnitude of the first FID sample point at each spatial location. Horizontal axis units for spectra are ppm.

in (II), where improved spatial localization and reduced spectral leakage can be observed at the expense of SNR. Unlike the synthetic case, in which the forward model was used to simulate the MRSI measurements, the ${ }^{1} \mathrm{H}$ reconstructions using the proposed method (III and IV) underscore the indispensability and efficacy of the TGV penalty. While the proposed method without the TGV regularization (III) is able to recover the general phantom geometry, the lack of additional spatial constraints render the method incapable of recovering uncontaminated spectra, and hence little is gained by way of spectral quality over standard Fourier reconstruction. Though null-space contributions are presupposed given the ill-posedness of the nonregularized reconstructions, a possibly incomplete measurement model, as discussed in greater detail further on, may explain the observed and seemingly exacerbated phase perturbations, as well as the inaptitude of the synthetic data in anticipating such artifacts. Alternatively, visual comparison between
(II) and (IV) encourages the notion that the TGV penalty provides the necessary conditioning to recover the true underlying spatio-spectral distribution. Indeed, though some influence from the outer compartment can be discerned in $\operatorname{IV}(a)-\operatorname{IV}(d)$, the severity is greatly attenuated over reconstructions (I-III).

It is important to consider throughout these analyses that the desired outcome in the synthetic MRSI dataset would be such that the final reconstructed dataset lie completely within a threedimensional subspace corresponding to the three ground-truth compartments, i.e., a SVD would yield only three nonzero singular values. Likewise, the ideal reconstructed ${ }^{1} \mathrm{H}$ MRSI dataset would lie within a 2-D subspace, corresponding to the inner and outer phantom compartments. In practice, however, such coveted outcomes are necessarily precluded by both the discrete nature of the data acquisition process, and to the ill-posedness of the reconstruction itself in the face of limited measurements. Nonetheless, the proposed method does produce reconstructions 
I

IFFT $(32 \times 32)$
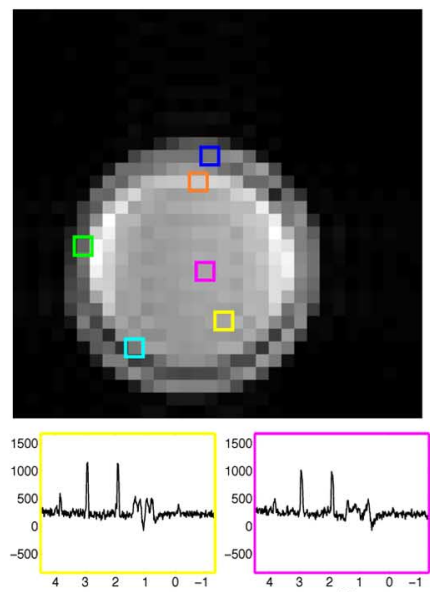

(a)

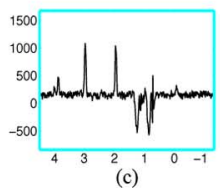

(c)

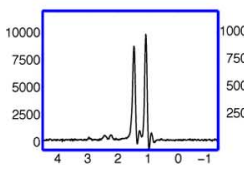

(e)

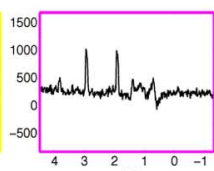

(b)

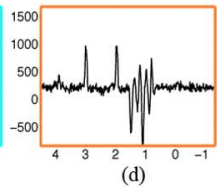

(d)

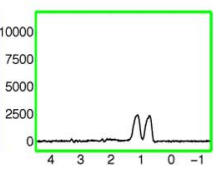

(f)
II

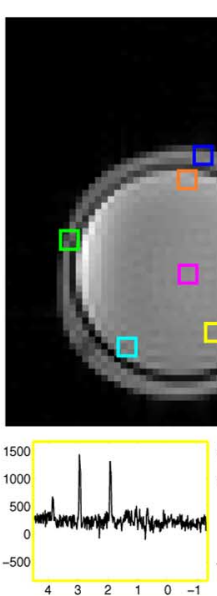

(a)

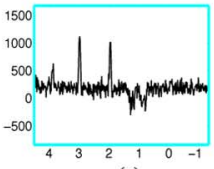

(c)

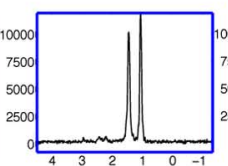

(e)
III

Proposed (no TGV)
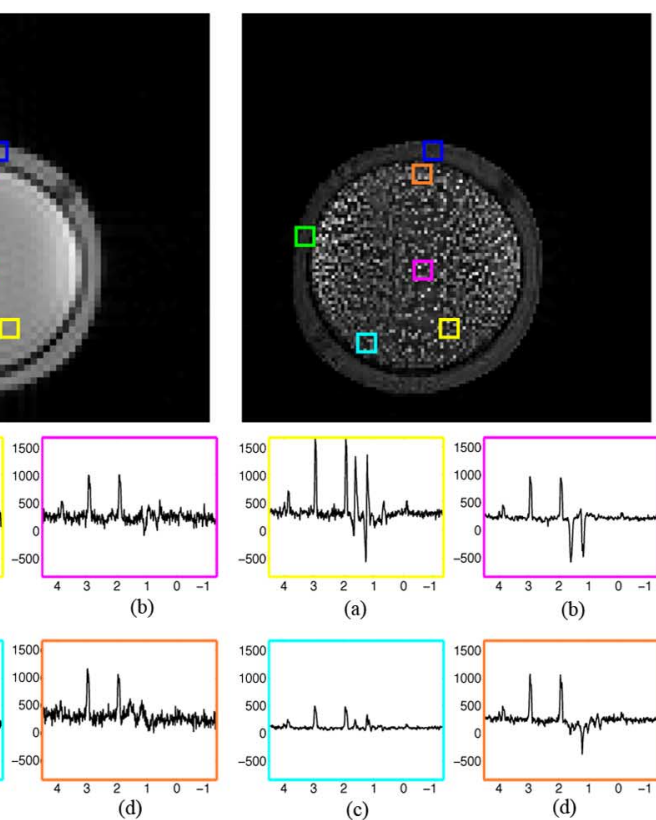

(d)

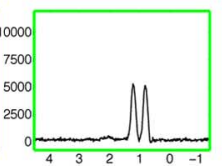

(f) (a)

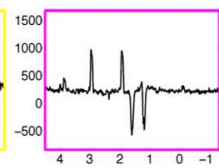

(b)

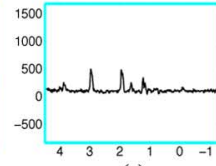

(c)

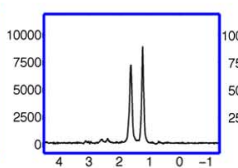

(e)

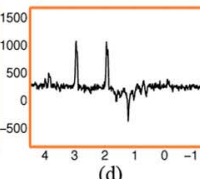

(d)

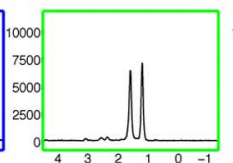

(f)
IV

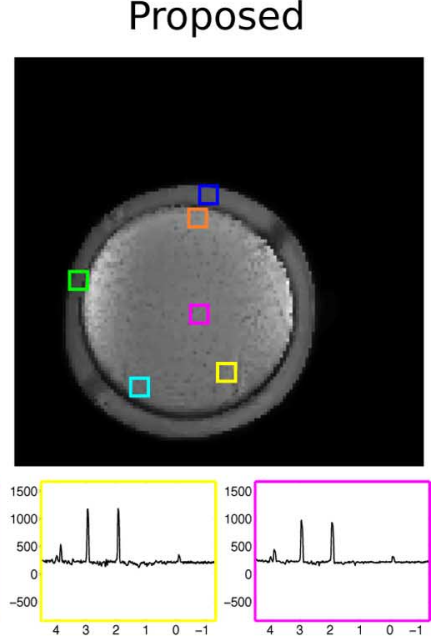

(a)

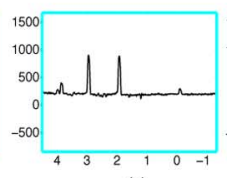

(c)

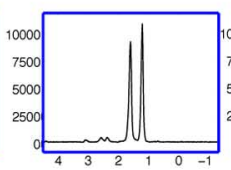

(e) (b)

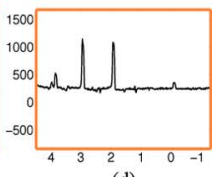

(d)

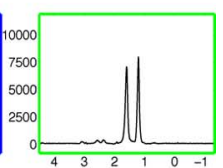

(f)

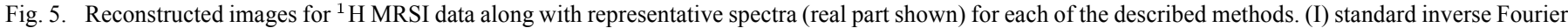

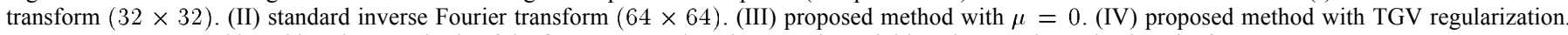
Images were generated by taking the magnitude of the first FID sample point at each spatial location. Horizontal axis units for spectra are ppm.

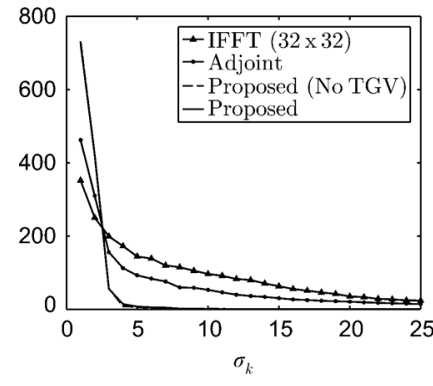

(a)

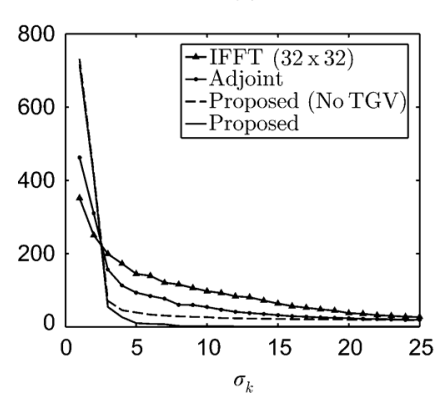

(c)

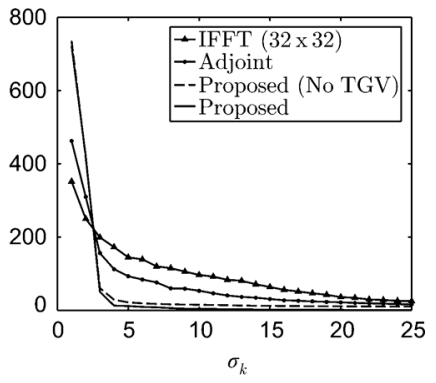

(b)

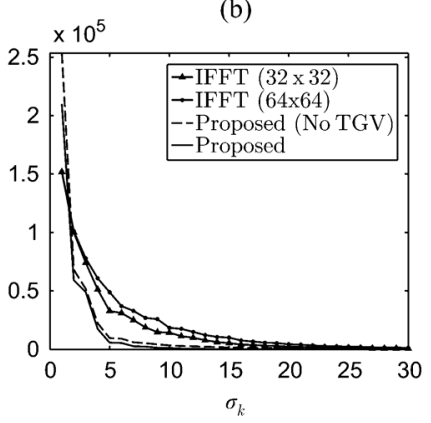

(d)

Fig. 6. Singular value spectra for reconstructed datasets. (a) Synthetic data (Case 1). (b) Synthetic data (Case 2). (c) Synthetic data (Case 3). (d) ${ }^{1}$ H MRSI phantom.

in which the energy is primarily captured by just a few singular values, thereby suggesting ultimately low dimensional representations (Fig. 6). Although this apportioning plainly promotes

the first three components in the synthetic case (which in turn best represent the ground truth spectral distribution), the situation clearly becomes more complicated in experimental settings [Fig. 6 (d)]. This is likely due to the presence of additional dynamic field perturbations, such as residual eddy currents caused by the gradient coils, which cannot be captured by a static $B_{0}$ inhomogeneity profile. Additionally, an inappropriate choice of $\triangle \mathrm{TE}$, insufficient SNR, or significant variations along the through-slice dimension can lead to an inaccurate measured $B_{0}$ map, which would thereby be unable to fully account even for the static inhomogeneities. Furthermore, variations in the $B_{1}$ field, additional off-resonance effects, motion-induced artifacts, or dynamic susceptibility variations due to flow phenomena in in vivo settings may introduce additional complexities which are unaccounted for by the basic acquisition model (8). A number of challenges therefore remain, coinciding with numerous active areas of research in MR spectroscopy and spectroscopic imaging. With regards to residual eddy current correction, several postprocessing methods have been developed, many of which use some type of reference signal (internal or external, e.g., [42], [43]), self-deconvolution [44], or even wavelets [45]. Most of these methods, however, were envisaged with regards to single voxel measurements, and their application to MRSI datasets would represent a significant increase in computation or acquisition time. In [46], a dynamic field camera [47], [48] monitors the field evolution during a given acquisition. The signal phase evolution is then estimated by fitting a set 
of solid spherical harmonic basis functions (up to third order) to the phase time courses of an array of NMR probes positioned around the sample.

Also worthy of mention is the fact that in the presented ${ }^{1} \mathrm{H}$ experiments, a relatively long echo time of $288 \mathrm{~ms}$ was chosen for the CSI acquisitions. Alternatively, short TE measurements are warranted in certain applications for which spectral information from shorter $T_{2}$ species is desired, which occasion additional encumbrances such as a less efficient water suppression, an increased lipid/metabolites ratio, and a more complex background spectral profile. Accordingly, commensurate compensatory mechanisms, for example, additional outer volume suppression (OVS) pulses to reduce the lipid signal, more robust water suppression techniques, or even explicit modeling of nuisance components, may be required in order to ensure accurate reconstruction.

A few words are merited with regards to the modeling assumptions behind the reconstruction framework. In essence, our scheme seeks a compromise between two broad generalizations about the problem geometry. The first, as has already been discussed, adheres to the validity of a bilinearly-representable functional form for the spatio-spectral distribution, whose dimensionality is ultimately low. We constrain our solutions to those matching this criteria by effectively placing an upper-bound on the data dimensionality, and restricting the reconstruction process to the circumscribed subspace. Though the nonconvexity of the problem undermines the uniqueness of the decomposition (in the absence of additional assumptions), the components themselves may undergo any energy-preserving reparametrization, which affords the user some flexibility with visualization. The SVD, for example, represents a parametrization enabling a user to view uncorrelated high-resolution spatial eigen-maps or eigen-spectra sorted by explained variance, though many other matrix factorizations may be conceived. The second, enforced via the total generalized variation-based spatial regularization, is that spatial components are represented as piecewise linear functions (piecewise constant with classical TV penalties), thereby restricting solutions to those that would be considered as physically plausible. Consequently, the regularization plays an important dual role which underlies the method's efficacy. In the absence of the regularization term, the reconstruction would consist entirely of linear operations, and the circumstances would revert back to those where explicit a priori models are needed in order to improve the problem conditioning. The nonlinearity of TV, however, intrinsically prescribes the geometrical framework for the high-resolution model, leaving remaining parameters to be estimated in the most data-consistent manner within the established subspace. Nonetheless, it is the synergy between the TV regularization and the high-resolution field map which furnishes the necessary problem conditioning for high-resolution MRSI reconstruction. Though TV is an important ingredient, its efficacy in extrapolating high-frequency information is diminished when considering higher degrees of sub-sampling. By not only stipulating the geometrical framework, but also injecting the implicit high-resolution information contained in the field map, the ill-posedness of the reconstruction is significantly abated. This obviates the spatial and spectral distribution equivalency posited by SLIM and its variants, and hence the need for supplementary structural MR images and the dilemma of optimally determining the compartments.

We note that similar spatial constraints were employed in [16] using classical TV penalties, though reconstructions were achieved on a lower resolution sampling grid. Minimization was also performed over the joint variable, $\mathbf{P}$ [see (10)]. While this formulation restores convexity, it compels the use of spatial regularization at each temporal sample point, thereby increasing the computational burden, especially if more sophisticated regularization or processing is later desired. Furthermore, the virtue of TV-based spatial regularization may diminish along the temporal dimension due to $T_{2}^{*}$ decay, whereby noise tends to dominate the signal. In contrast, the bilinear model confines the needed spatial regularization operations to those maps lying within the working subspace of dimensionality $K$, where often times $K \ll \min \{M, T\}$. Another important distinction is that whereas [16] limits the admissible spectra to sparse combinations of Diracs and polynomials, we refrain from such explicit spectral parametrizations in order to observe the full temporal dynamics of the signal. As a final merit, it should be noted that our method remains fully compatible with non-Cartesian sampling strategies, thereby broadening its suitability for a wide array of MRSI acquisition types.

From a clinical standpoint, the potential utility of such data-driven spectral localization procedures is extensive. One particularly befitting application, and one for which MRSI has gained notoriety as a promising adjuvant diagnostic or prognostic tool, is in the study of brain tumors (for a review, see [49]). For example, while contrast-enhanced structural MRI is a powerful and noninvasive diagnostic aid, its specificity for differentiating between tumor types, neoplastic and nonneoplastic lesions, and identifying regions of active tumor is minimal [50]-[54]. A number of studies have highlighted the discriminating capability of MRSI in differential diagnostics, emphasizing its capacity for elucidating tumor heterogeneity [55]-[59]. Additionally, some studies on high-grade gliomas reported that the extent of the spatial maps of metabolic abnormality derived from MRSI measurements often exceeded the pathological volume defined on the accompanying structural MR images [60]-[63], and that correlations with tumor recurrence following radiosurgery could be observed [64]-[66]. Such findings consequently impugn the adequacy of structurally-defined spatial models such as SLIM, which can be even further confounded by the difficulty in segmenting the types of diffuse and poorly-defined border regions characteristic of high-grade gliomas [67], [68]. The use of more data-driven approaches therefore serves as an attractive alternative in extracting delineating spatial information offered by MRSI, which can be crucial in determining appropriate patient-specific treatment strategies.

\section{CONCLUSION AND FutURE WORK}

In this work, we have developed a novel approach for the reconstruction of MRSI data which overcomes a number of limitations associated with standard inverse Fourier methods, most notably spectral leakage artifacts and rectangular pixel sizes, and requires only a $B_{0}$ inhomogeneity map as an additional 
measurement. What's more, the method does not require an explicit spatial model, perhaps the most tendentious aspect of the SLIM approach, which thereby allows for the analysis of MRSI data on a much more general level. We demonstrated improved reconstructions in both synthetic and ${ }^{1} \mathrm{H}$ MRSI phantom studies over their standard inverse Fourier counterparts. Currently, our method is able to effectively nullify the impact of static $B_{0}$ inhomogeneities only, and the effect of dynamic phase perturbations will be studied in future work. With the aim to further improve reconstruction quality, we also plan to extend our approach to multi-slice MRSI acquisitions, and to explore alternative sampling strategies tailored to our reconstruction formulation.

\section{ACKNOWLEDGMENT}

The authors would like to thank Dr. A. Henning and T. Kirchner at the Institute for Biomedical Engineering, ETH Zurich for their helpful comments and suggestions. The authors would also like to thank the anonymous reviewers, whose insightful comments significantly improved the quality of the manuscript.

\section{REFERENCES}

[1] J. Suhy, K. Laxer, A. Capizzano, P. Vermathen, G. Matson, N. Barbaro, and M. Weiner, " $1 \mathrm{H}$ MRSI predicts surgical outcome in MRI-negative temporal lobe epilepsy," Neurology, vol. 58, no. 5, pp. 821-823, 2002.

[2] G. S. Aaen, B. A. Holshouser, C. Sheridan, C. Colbert, M. McKenney, D. Kido, and S. Ashwal, "Magnetic resonance spectroscopy predicts outcomes for children with nonaccidental trauma," Pediatrics, vol. 125, pp. 295-303, Feb. 2010

[3] T. R. Brown, B. M. Kincaid, and K. Ugurbil, "NMR chemical shift imaging in three dimensions," Proc. Nat. Acad. Sci. USA, vol. 79, no. 11, pp. 3523-3526, 1982.

[4] A. Greiser and M. v. Kienlin, "Efficient k-space sampling by density-weighted phase-encoding," Magn. Reson. Med., vol. 50, no. 6, pp. $1266-1275,2003$

[5] J. W. Hugg, A. A. Maudsley, M. W. Weiner, and G. B. Matson, "Comparison of k-space sampling schemes for multidimensional MR spectroscopic imaging," Magn. Reson. Med., vol. 36, pp. 469-473, Sep. 1996.

[6] C.-M. Tsai and D. G. Nishimura, "Reduced aliasing artifacts using variable-density k-space sampling trajectories," Magn. Reson. Med., vol. 43, no. 3, pp. 452-458, 2000.

[7] S. Uribe, A. Guesalaga, R. Mir, M. Guarini, and P. Irarrázaval, "A 3D trajectory for undersampling k-space in MRSI applications," Magn. Reson. Imag., vol. 25, no. 3, pp. 350-358, 2007.

[8] D.-H. Kim, R. Henry, and D. M. Spielman, "Fast multivoxel two-dimensional spectroscopic imaging at 3T," Magn. Reson. Imag., vol. 25, no. 8, pp. 1155-1161, 2007.

[9] S. Sarkar, K. Heberlein, and X. Hu, "Truncation artifact reduction in spectroscopic imaging using a dual-density spiral k-space trajectory," Magn. Reson. Imag., vol. 20, no. 10, pp. 743-757, 2002.

[10] R. Pohmann, E. Rommel, and M. v. Kienlin, "Beyond k-Space: Spectral localization using higher order gradients," J. Magn. Reson., vol. 141, no. 2, pp. 197-206, 1999.

[11] K. P. Pruessmann, M. Weiger, M. B. Scheidegger, and P. Boesiger, "Sense: Sensitivity encoding for fast MRI," Magn. Reson. Med., vol. 42, pp. 952-962, Nov. 1999.

[12] X. Hu, D. N. Levin, P. C. Lauterbur, and T. Spraggins, "SLIM: Spectral localization by imaging," Magn. Reson. Med., vol. 8, pp. 314-322, Nov. 1988.

[13] Z. P. Liang and P. C. Lauterbur, "A generalized series approach to MR spectroscopic imaging," IEEE Trans. Med. Imag., vol. 10, no. 2, pp. 132-137, Jun. 1991.

[14] I. Khalidov, D. V. DeVille, M. Jacob, F. Lazeyras, and M. Unser, "BSLIM: Spectral localization by imaging with explicit $B_{0}$ field inhomogeneity compensation," IEEE Trans. Med. Imag., vol. 26, no. 7, pp. 990-1000, Jul. 2007.

[15] M. Jacob, X. Zhu, A. Ebel, N. Schuff, and Z.-P. Liang, "Improved model-based magnetic resonance spectroscopic imaging," IEEE Trans. Med. Imag., vol. 26, no. 10, pp. 1305-1318, Oct. 2007.
[16] R. Eslami and M. Jacob, "Robust reconstruction of MRSI data using a sparse spectral model and high resolution MRI priors," IEEE Trans. Med. Imag., vol. 29, 6, pp. 1297-1309, Jun. 2010.

[17] P. Jezzard and R. S. Balaban, "Correction for geometric distortion in echo planar images from B0 field variations," Magn. Reson. Med., vol. 34, pp. 65-73, Jul. 1995.

[18] H. Pedersen, S. Kozerke, S. Ringgaard, K. Nehrke, and W. Y. Kim, "K-tPCA: Temporally constrained k-t BLAST reconstruction using principal component analysis," Magn. Reson. Med., vol. 62, pp. 706-716, Sep. 2009.

[19] Z.-P. Liang, "Spatiotemporal imaging with partially separable functions," in Proc. 4th IEEE Int. Symp. Biomed. Imag.: From Nano to Macro, Apr. 2007, pp. 988-991.

[20] J. Haldar and Z.-P. Liang, "Spatiotemporal imaging with partially separable functions: A matrix recovery approach," in Proc. IEEE Int. Symp. Biomed. Imag.: From Nano to Macro, Apr. 2010, pp. 716-719.

[21] S. Lingala, Y. Hu, E. DiBella, and M. Jacob, "Accelerated dynamic MRI exploiting sparsity and low-rank structure: k-t SLR," IEEE Trans. Med. Imag., vol. 30, no. 5, pp. 1042-1054, May 2011.

[22] P. Sajda, S. Du, T. Brown, R. Stoyanova, D. Shungu, X. Mao, and L. Parra, "Nonnegative matrix factorization for rapid recovery of constituent spectra in magnetic resonance chemical shift imaging of the brain," IEEE Trans. Med. Imag., vol. 23, no. 12, pp. 1453-1465, Dec. 2004.

[23] Z.-P. Liang and P. Lauterbur, "Constrained imaging: Overcoming the limitations of the Fourier series," IEEE Eng. Med. Biol. Mag., vol. 15, pp. 126-132, Sep./Oct. 1996

[24] E. M. Haacke, Z. P. Liang, and S. H. Izen, "Constrained reconstruction: A super resolution, optimal signal-to-noise alternative to the Fourier transform in magnetic resonance imaging," Med. Phys., vol. 16, pp. 388-397, 1989.

[25] K. Wear, K. Myers, S. Rajan, and L. Grossman, "Constrained reconstruction applied to 2-D chemical shift imaging," IEEE Trans. Med. Imag., vol. 16, no. 5, pp. 591-597, Oct. 1997.

[26] L. I. Rudin, S. Osher, and E. Fatemi, "Nonlinear total variation based noise removal algorithms," Physica D: Nonlinear Phenomena, vol. 60, pp. 259-268, 1992.

[27] K. T. Block, M. Uecker, and J. Frahm, "Suppression of MRI truncation artifacts using total variation constrained data extrapolation," Int. J. Biomed. Imag., vol. 2008, pp. 184123-184123, 2008.

[28] A. Beck and M. Teboulle, "Fast gradient-based algorithms for constrained total variation image denoising and deblurring problems," IEEE Trans. Image Process., vol. 18, no. 11, pp. 2419-2434, Nov. 2009.

[29] F. Huang, Y. Chen, G. R. Duensing, J. Akao, A. Rubin, and C. Saylor, "Application of partial differential equation-based inpainting on sensitivity maps," Magn. Reson. Med., vol. 53, no. 2, pp. 388-397, 2005.

[30] M. Lustig, D. Donoho, and J. M. Pauly, "Sparse MRI: The application of compressed sensing for rapid MR imaging," Magn. Reson. Med., vol. 58, pp. 1182-1195, Dec. 2007.

[31] A. Chambolle and T. Pock, "A first-order primal-dual algorithm forConvex problems with applications to imaging," J. Math. Imag. Vis., vol. 40, pp. 120-145, May 2011.

[32] F. Knoll, K. Bredies, T. Pock, and R. Stollberger, "Second order total generalized variation (TGV) for MRI," Magn. Reson. Med., vol. 65, no. 2, pp. 480-491, 2011.

[33] Y. Hu and M. Jacob, "Higher degree total variation (HDTV) regularization for image recovery," IEEE Trans. Image Process., vol. 21, no. 5, pp. 2559-2571, May 2012.

[34] Z. Dogan, S. Lefkimmiatis, A. Bourquard, and M. Unser, "A secondorder extension of TV regularization for image deblurring," in Proc. 18th IEEE Int. Conf. Image Process., Sep. 2011, pp. 705-708.

[35] T. Chan, A. Marquina, and P. Mulet, "High-order total variation-based image restoration," SIAM J. Sci. Comput., vol. 22, no. 2, pp. 503-516, 2000.

[36] M. Yaghoobi, T. Blumensath, and M. Davies, "Dictionary learning for sparse approximations with the majorization method," IEEE Trans. Signal Process., vol. 57, no. 6, pp. 2178-2191, Jun. 2009.

[37] K. Kreutz-Delgado, J. F. Murray, B. D. Rao, K. Engan, T.-W. Lee, and T. J. Sejnowski, "Dictionary learning algorithms for sparse representation," Neural Comput., vol. 15, pp. 349-396, Feb. 2003.

[38] S. Lingala and M. Jacob, "A blind compressive sensing frame work for accelerated dynamic MRI," in Proc. 9th IEEE Int. Symp. Biomed. Imag., May 2012, pp. 1060-1063.

[39] M. Afonso, J. Bioucas-Dias, and M. Figueiredo, "Fast image recovery using variable splitting and constrained optimization," IEEE Trans. Image Process., vol. 19, no. 9, pp. 2345-2356, Sep. 2010. 
[40] Y. Nesterov, "A method for solving the convex programming problem with convergence rate $\mathcal{O}\left(1 / k^{2}\right)$," Dokl. Akad. Nauk SSSR, vol. 269, no. 3 , pp. $543-547,1983$.

[41] T. P. Minka, "Automatic choice of dimensionality for PCA," Adv. Neural Inform. Process. Syst., vol. 13, no. 514, pp. 598-604, 2001.

[42] U. Klose, "In vivo proton spectroscopy in presence of eddy currents," Magn. Reson. Med., vol. 14, pp. 26-30, Apr. 1990.

[43] J. R. Roebuck, D. O. Hearshen, M. O’Donnell, and T. Raidy, “Correction of phase effects produced by eddy currents in solvent suppressed 1HCSI," Magn. Reson. Med., vol. 30, no. 3, pp. 277-282, 1993.

[44] A. A. Maudsley, "Spectral lineshape determination by self-deconvolution," J. Magn. Reson. B, vol. 106, pp. 47-57, Jan. 1995.

[45] D. Barache, J.-P. Antoine, and J.-M. Dereppe, "The continuous wavelet transform, an analysis tool for NMR spectroscopy," J. Magn. Reson., vol. 128, no. 1, pp. 1-11, 1997.

[46] B. J. Wilm, C. Barmet, M. Pavan, and K. P. Pruessmann, "Higher order reconstruction for MRI in the presence of spatiotemporal field perturbations," Magn. Reson. Med., vol. 65, no. 6, pp. 1690-1701, 2011

[47] C. Barmet, B. Wilm, M. Pavan, and K. Pruessmann, "A third-order field camera with microsecond resolution for MR system diagnostics," in Proc. 7th Sci. Meet. Exhibit., 2009.

[48] C. Barmet, B. Wilm, M. Pavan, G. Katsikatsos, J. Geupp, G. Mens, and K. Pruessmann, "Concurrent higher-order field monitoring for routine head MRI: An integrated heteronuclear setup," in Proc. 18th Sci. Meet. Exhibit., 2010, pp. 216-216.

[49] A. Horská and P. B. Barker, "Imaging of brain tumors: MR spectroscopy and metabolic imaging," Neuroimag. Clin. N. Am., vol. 20, pp. 293-310, Aug. 2010.

[50] A. M. Omuro, C. C. Leite, K. Mokhtari, and J.-Y. Delattre, "Pitfalls in the diagnosis of brain tumours," Lancet Neurol., vol. 5, no. 11, pp. 937-948, 2006.

[51] R. Hourani, L. J. Brant, T. Rizk, J. D. Weingart, P. B. Barker, and A. Horska, "Can proton MR spectroscopic and perfusion imaging differentiate between neoplastic and nonneoplastic brain lesions in adults?," AJNR Am. J. Neuroradiol., vol. 29, pp. 366-372, Feb. 2008.

[52] G. S. Payne and M. O. Leach, "Applications of magnetic resonance spectroscopy in radiotherapy treatment planning," Br. J. Radiol., vol. 79, no. 1, pp. 16-26, Sep. 2006.

[53] F. Earnest, P. J. Kelly, B. W. Scheithauer, B. A. Kall, T. L. Cascino, R. L. Ehman, G. S. Forbes, and P. L. Axley, "Cerebral astrocytomas: Histopathologic correlation of MR and CT contrast enhancement with stereotactic biopsy," Radiology, vol. 166, pp. 823-827, Mar. 1988

[54] D. Kondziolka, L. D. Lunsford, and A. J. Martinez, "Unreliability of contemporary neurodiagnostic imaging in evaluating suspected adult supratentorial (low-grade) astrocytoma," J. Neurosurg., vol. 79, pp. 533-536, Oct. 1993.

[55] A. J. Martin, H. Liu, W. A. Hall, and C. L. Truwit, "Preliminary assessment of turbo spectroscopic imaging for targeting in brain biopsy," AJNR Am. J. Neuroradiol., vol. 22, pp. 959-968, May 2001.

[56] A. Stadlbauer, S. Gruber, C. Nimsky, R. Fahlbusch, T. Hammen, R. Buslei, B. Tomandl, E. Moser, and O. Ganslandt, "Preoperative grading of gliomas by using metabolite quantification with high-spatial-resolution proton MR spectroscopic imaging," Radiology, vol. 238, pp. 958-969, Mar. 2006.
[57] M. C. Preul, Z. Caramanos, D. L. Collins, J. G. Villemure, R. Leblanc, A. Olivier, R. Pokrupa, and D. L. Arnold, "Accurate, noninvasive diagnosis of human brain tumors by using proton magnetic resonance spectroscopy," Nat. Med., vol. 2, pp. 323-325, Mar. 1996.

[58] A. R. Tate, C. Majós, A. Moreno, F. A. Howe, J. R. Griffiths, and C. Arús, "Automated classification of short echo time in in vivo $1 \mathrm{H}$ brain tumor spectra: A multicenter study," Magn. Reson. Med., vol. 49, no. 1, pp. 29-36, 2003.

[59] A. DiCostanzo, T. Scarabino, F. Trojsi, G. M. Giannatempo, T. Popolizio, D. Catapano, S. Bonavita, N. Maggialetti, M. Tosetti, U. Salvolini, V. A. d'Angelo, and G. Tedeschi, "Multiparametric 3TMR approach to the assessment of cerebral gliomas: Tumor extent and malignancy," Neuroradiology, vol. 48, pp. 622-631, Sep. 2006.

[60] A. Stadlbauer, E. Moser, S. Gruber, R. Buslei, C. Nimsky, R. Fahlbusch, and O. Ganslandt, "Improved delineation of brain tumors: an automated method for segmentation based on pathologic changes of 1H-MRSI metabolites in gliomas," NeuroImage, vol. 23, no. 2, pp. 454-461, 2004.

[61] A. Pirzkall, T. R. McKnight, E. E. Graves, M. P. Carol, P. K. Sneed, W. W. Wara, S. J. Nelson, L. J. Verhey, and D. A. Larson, "MR-spectroscopy guided target delineation for high-grade gliomas," Int. J. Radiat. Oncol. Biol. Phys., vol. 50, no. 4, pp. 915-928, 2001.

[62] O. Ganslandt, A. Stadlbauer, R. Fahlbusch, K. Kamada, R. Buslei, I. Blumcke, E. Moser, and C. Nimsky, "Proton magnetic resonance spectroscopic imaging integrated into image-guided surgery: Correlation to standard magnetic resonance imaging and tumor cell density," Neurosurgery, vol. 56, pp. 291-298, Apr. 2005.

[63] T. R. McKnight, M. H. v. Bussche, D. B. Vigneron, Y. Lu, M. S. Berger, M. W. McDermott, W. P. Dillon, E. E. Graves, A. Pirzkall, and S. J. Nelson, "Histopathological validation of a three-dimensional magnetic resonance spectroscopy index as a predictor of tumor presence," J. Neurosurg., vol. 97, pp. 794-802, Oct. 2002.

[64] A. A. Chan, A. Lau, A. Pirzkall, S. M. Chang, L. J. Verhey, D. Larson, M. W. McDermott, W. P. Dillon, and S. J. Nelson, "Proton magnetic resonance spectroscopy imaging in the evaluation of patients undergoing gamma knife surgery for grade IV glioma," J. Neurosurg., vol. 101, pp. 467-475, Sep. 2004.

[65] E. E. Graves, S. J. Nelson, D. B. Vigneron, C. Chin, L. Verhey, M. McDermott, D. Larson, P. K. Sneed, S. Chang, M. D. Prados, K. Lamborn, and W. P. Dillon, "A preliminary study of the prognostic value of proton magnetic resonance spectroscopic imaging in gamma knife radiosurgery of recurrent malignant gliomas," Neurosurgery, vol. 46, pp. 319-326, Feb. 2000

[66] E. E. Graves, S. J. Nelson, D. B. Vigneron, L. Verhey, M. McDermott, D. Larson, S. Chang, M. D. Prados, and W. P. Dillon, "Serial proton MR spectroscopic imaging of recurrent malignant gliomas after gamma knife radiosurgery," AJNR Am. J. Neuroradiol., vol. 22, pp. 613-624, Apr. 2001

[67] S. J. Nelson, "Multivoxel magnetic resonance spectroscopy of brain tumors," Mol. Cancer Ther., vol. 2, pp. 497-507, May 2003.

[68] M. A. Weber, F. L. Giesel, and B. Stieltjes, "MRI for identification of progression in brain tumors: From morphology to function," Expert Rev. Neurother., vol. 8, pp. 1507-1525, Oct. 2008. 